\title{
OBJECTIVE QUALITY CONTROL PROGRAM FOR MEDICAL ULTRASOUND SCANNING
}

\author{
Amr A. Sharawy ${ }^{1}$, Kamel. K. Mohammed ${ }^{1}$, and Mohamed Aouf ${ }^{2}$ \\ ${ }^{1}$ Biomedical Engineering Department, Cairo University, Giza 12613, Egypt \\ ${ }^{2}$ Biomedical Engineering Department, Higher Technological Institute, Tenth of Ramadan City, Egypt
}

\begin{abstract}
Ultrasound imaging of a focal lesion of low contrast against background tissue necessitates maximum spatial and contrast sensitivity resulting in non-specular reflection, predisposing these images to degradation due to speckle. In recent years, diagnostic ultrasound image quality has greatly improved due to advances in system hardware and software. Developments in transducer design have resulted in transducers with greater bandwidth and sensitivity. Quality features to be tested are spatial resolution, contrast resolution and lesion signal-tonoise ratio. These features are explained and examples are shown.
\end{abstract}

\section{KEY WORDS: Contrast, Resolution, Speckle Noise, Phantom, Ultrasound Imaging.}

\section{INTRODUCTION}

Evolutions in the field of diagnostic ultrasound (e.g. the worldwide market revenues in ultrasound imaging are expected to grow from about $\$ 4$ billion in 2012 to $\$ 5$ billion by 2019)

[1] have led to increased use of this modality in many clinical applications. Ultrasound is often considered the preferred imaging modality because of its ability to provide continuous, real time images without the risk of ionizing radiation and at a lower cost than a Computed Tomography (CT) or Magnetic Resonance Imaging (MRI) scan. The accuracy of sonography for the diagnosis of hepatocellular carcinoma (HCC) is considered to be high [2]. However, in patients with viral liver cirrhosis or chronic viral hepatitis, the echo pattern of the liver parenchyma is rough and nodular. As a result, detection of a small malignant nodule among multiple regenerative nodules is very difficult [3]. This represents a limitation of sonography for the early detection of HCC. Because the final image is the basis for diagnostic decisions, the image quality produced by a scanner provides the most important information in testing scanner quality assessment. Measuring the detectability of small well-characterized, lowcontrast lesions is important for quantifying the image quality of a system. The quality control of various ultrasound units is performed on the basis of detection of focal lesions against background tissue because this detection necessitates high spatial resolution and contrast sensitivity. Several methods for calculating the image quality have been developed for assisting in the design and development of various ultrasound machines.

A final aspect of quality assessment procedures and protocols that have been published so far [4-7] is the subjective nature of the testing procedures. For this reason, the testing results become strongly dependent on the chosen settings of the equipment and on the visual interpretation of displayed information during the testing, i.e. the observer is part of the procedure. Although the recent paper by Sugawara et al. [8] is based on objective assessment, a serious limitation is the use of gray level rather than relative echo level (in $\mathrm{dB}$ ), as was proposed by Thijssen et al. [9-10], which is incorporated also in new IEC standards being developed. More recently, the Thijssen et al. have developed a protocol [11] based on quality assessment by software algorithms applied to stored digital ultrasound images, while using reproducible equipment settings. The aim of this contribution is to apply these methods in a comparison of different ultrasound scanners from different vendors. 


\section{MATERIAL AND METHODES \\ Equipment:}

There were Solo Compact Ultrasound Imaging System (International Biomedical Engineering Technologies, Egypt) and Fukuda.

\section{Tissue mimicking phantom:}

The quality control of ultrasound was performed by using a tissue mimicking "phantom" Computerized Imaging Reference Systems (CIRS) Model 040 phantom consisting of solid elastic material called Zerdine that is not affected by changes in temperature. Additionally, this phantom contains several targets arranged in special patterns. The attenuation of the base material are similar to human liver tissue (speed of sound $1540 \mathrm{~m} / \mathrm{s}$; attenuation coefficient 0.5 to $0.7 \mathrm{~dB} / \mathrm{cm}$.MHz.). These properties are critical for imaging because they simulate focal lesions against background tissue used for quality control and testing of ultrasound transducer.

\section{Image Quality Tests:}

The image quality parameters that were measured in the study were: 1 . axial resolution; 2 . lateral resolution; 3. contrast resolution; 4. contrast sensitivity; and 5. dynamic range.

These image quality test parameters were chosen for testing the ultrasound scanner to obtain a full evaluation of its imaging capabilities, as recommended by a number of professional bodies. Such evaluation performed subjectively by using dedicated software applications for automated image evaluation and reporting (as, QA4US, Thijssen et al [11]).

\section{Contrast resolution:}

The contrast resolution is represented by the gamma of the system. Some tissue mimicking test objects (e.g. ATS Labs, Bridgeport, CT) contain cylinders with fixed diameter and scattering levels different from the surrounding material ("background"). By measuring the mean gray level of each of the cylinders (in cross-section visible as a disc) and plotting these against its known nominal contrast value in $\mathrm{dB}$, a linear regression can be made. The slope of this line yields the "gamma" of the systems in gray levels per $\mathrm{dB}$ and the $\mathrm{dB}$ range corresponding to gray levels from 0 to 255 yields the contrast dynamic range [11].

\section{Contrast sensitivity:}

The contrast sensitivity is defined as the smallest echo level contrast, with respect to the surrounding scattering medium, of a lesion of a certain size that can be detected. In the past, human observer experiments were carried out in which the contrast threshold was estimated for lesions of varying size [12], [13]. These experiments yielded a contrast sensitivity measure of the combination imaging system-human observer. The purpose of the measurements in this paper is to characterize only the imaging system. In practice, it is useful to assess the lesion signal-to-noise ratio, SNRL [14], [15], [16]:

$\operatorname{SNR}_{L}=\frac{\left|\left\langle\mu_{2}\right\rangle-\left\langle\mu_{1}\right\rangle\right|}{\sqrt{\sigma_{\mu_{2}}^{2}+\sigma_{\mu_{1}}^{2}}}$

Where $\left\langle\boldsymbol{\mu}_{\mathbf{2}}\right\rangle$ and $\left\langle\boldsymbol{\mu}_{\mathbf{1}}\right\rangle$ are mean grey level within a circular area of surrounding (background) tissue (2), and of lesion (1), averaged over the ensemble of images from independent scans. and $\sigma_{\mu_{1}}^{2}$ are variance of mean grey level of area of background (1) and of lesion (2) [11].

\section{Spatial resolution}

The geometrical resolution is defined as the axial, or lateral, FWHM, i.e. -6dB width, of the image of a small object. Axial and lateral resolution were measured by the automated image analysis software by drawing a region-of-interest (ROI) around the cross sectional image of the nylon filament targets and measuring the $-6 \mathrm{~dB}$ width above the surrounding background of the filament in both the axial and lateral directions [7]. 


\section{RESULTS}

\section{Contrast Resolution:}

For this measurement four cross-sectional images of each cylinder were acquired and analyzed with equal size regions of interest (ROIs). The mean and standard deviation of the grey levels within a fixed circular area fitting into the displayed discs is estimated for each object. This circle is also used for selecting the background discs. These values are plotted against the nominal contrast values in $\mathrm{dB}$, as shown in Fig. 1.

The estimated contrast resolution (i.e., gamma) and the overall dynamic range are then estimated. The gamma is used to estimate $\mathrm{dB}$ echo levels from measured gray levels in several image quality characteristics. The dynamic range determines the range of intensities to be displayed: a large dynamic range tends to reduce contrast resolution because the grey scale is 'shared' by a wider range of echo intensities. Therefore, Solo scanner better than Fucuda scanner because dynamic range of the Solo scanner higher than dynamic range of the Fucuda scanner.
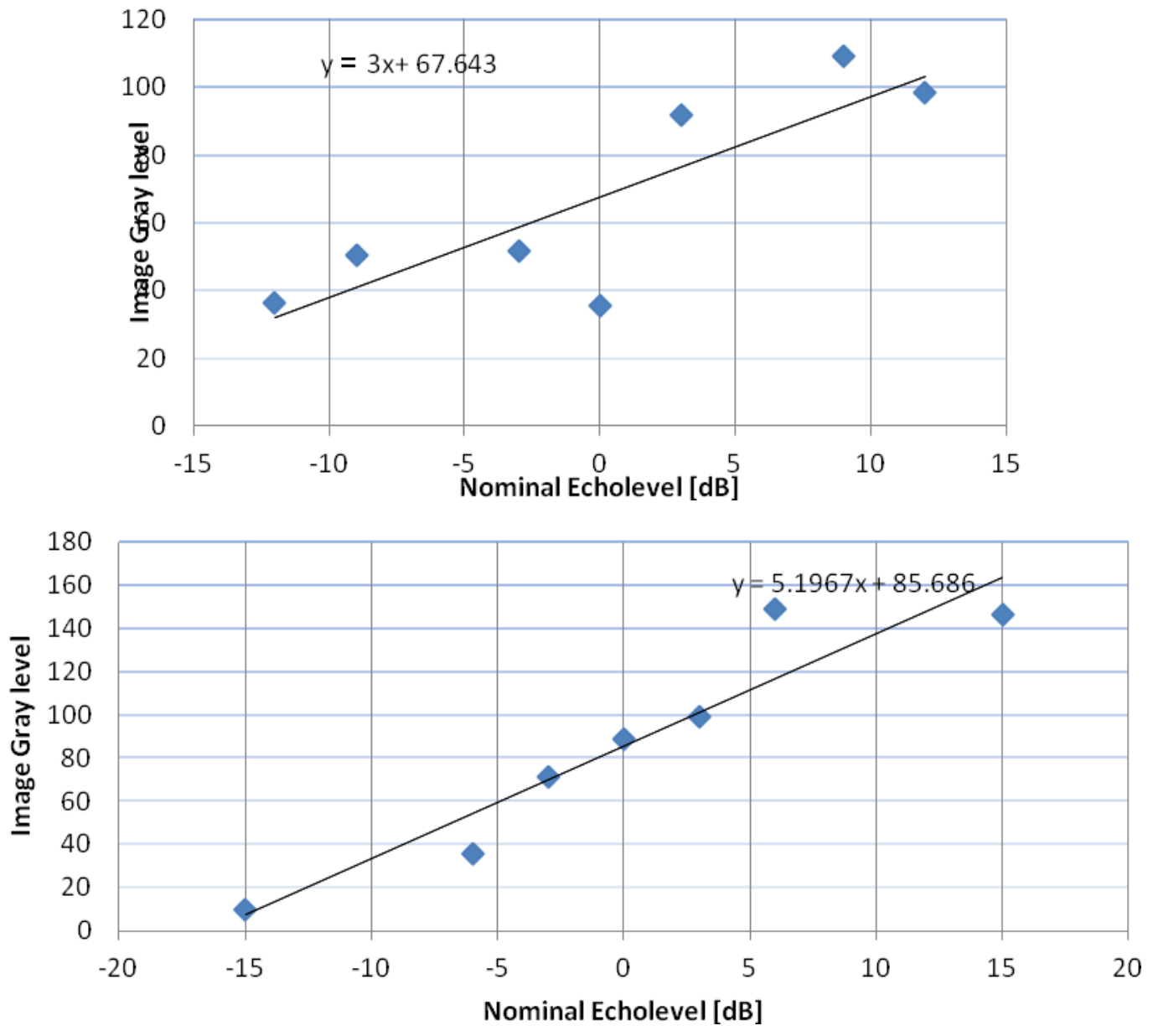

Figure 1: Plot of mean gray level of lesions (average of five measurements and standard deviation; left abscissa) vs. nominal contrast values of scattering lesions. Linear regression yields contrast resolution (gamma = number of gray levels/dB) and echo level dynamic range (\# $\mathrm{dB}$ for full range $(0-255))$. Top: contrast resolution curves for equipment 1 "Fucuda" and bottom contrast resolution curves for equipment 2 "Solo".

\section{Contrast sensitivity:}

The lesion signal-to-noise ratio, $\mathrm{SNR}_{\mathrm{L}}$ was calculated. The results for equipment 1 are shown in Table 1 and the results for equipment 2 are shown in Table 2. However, a systematic difference between the Fukuda and Solo modes seems to be exist. SNR $\mathrm{L}_{\mathrm{increases}}$ as speckle noise decreases, 
generally resulting in maximum contrast. Contrast model for ultrasound B-mode images consists of light regions (i.e. regions have maximum echo amplitude) and dark regions (i.e. regions have minimum echo amplitude), minimum quality images tend to exhibit a minimum difference between light and dark regions, and therefore it is difficult to observe objects as compared with maximum contrast images.

Table 1: Contrast resolution, dynamic range and spatial resolution for Fukuda.

\begin{tabular}{|c|c|c|c|c|}
\cline { 2 - 5 } \multicolumn{1}{c|}{} & $\begin{array}{c}\text { Image } \\
1\end{array}$ & \begin{tabular}{c} 
Image \\
\multicolumn{1}{c|}{}
\end{tabular} & $\begin{array}{c}\text { Image } \\
3\end{array}$ & $\begin{array}{c}\text { Image } \\
4\end{array}$ \\
\hline $\begin{array}{c}\text { Contrast } \\
\text { Resolution } \\
\text { of Fucuda }\end{array}$ & \multicolumn{3}{|c|}{ Gamma = [echo levels/dB] } \\
\hline $\begin{array}{c}\text { Dynamic } \\
\text { Range }\end{array}$ & \multicolumn{2}{|c|}{ Dynamic Range = 86 } \\
\hline $\begin{array}{c}\text { SNR } \\
\text { Lateral } \\
\text { Resolution } \\
\text { at 6 cm of } \\
\text { Fucuda }\end{array}$ & 0.33 & 0.20 & 0.42 & 0.17 \\
\hline $\begin{array}{c}\text { Axial } \\
\text { Resolution } \\
\text { at } 6 \text { cm of } \\
\text { Fucuda }\end{array}$ & 0.24 & 0.07 & 0.66 & 0.07 \\
\hline $\begin{array}{c}\text { Lateral } \\
\text { Resolution } \\
\text { at 8 cm of } \\
\text { Fucuda }\end{array}$ & 0.36 & 0.49 & 0.65 & 0.52 \\
\hline $\begin{array}{c}\text { Axial } \\
\text { Resolution } \\
\text { at 8 cm of } \\
\text { Fucuda }\end{array}$ & 0.46 & 0.56 & 0.28 & 0.54 \\
\hline
\end{tabular}

Table 2: Contrast resolution, dynamic range and spatial resolution for Solo.

\begin{tabular}{|c|c|c|c|c|}
\cline { 2 - 5 } \multicolumn{1}{c|}{} & \begin{tabular}{c} 
Image \\
\multicolumn{1}{c|}{}
\end{tabular} & $\begin{array}{c}\text { Image } \\
2\end{array}$ & $\begin{array}{c}\text { Image } \\
3\end{array}$ & $\begin{array}{c}\text { Image } \\
4\end{array}$ \\
\hline $\begin{array}{c}\text { Contrast } \\
\text { Resolution } \\
\text { for Solo }\end{array}$ & \multicolumn{2}{|c|}{ Gamma $=5.2$ [echo levels/dB] } \\
\hline $\begin{array}{c}\text { Dynamic } \\
\text { Range }\end{array}$ & \multicolumn{2}{|c|}{ Dynamic Range $=49$} & 0.36 \\
\hline SNR & \multicolumn{2}{|c|}{ Signal to noise ratio = 2.7 } \\
\hline $\begin{array}{c}\text { Lateral } \\
\text { Resolution } \\
\text { at 6 cm } \\
\text { for Solo }\end{array}$ & 0.18 & 0.20 & 0.32 & 0.73 \\
\hline $\begin{array}{c}\text { Axial } \\
\text { Resolution } \\
\text { at } 6 \mathrm{~cm} \\
\text { for Solo }\end{array}$ & 0.10 & 0.08 & 0.14 & 0.23 \\
\hline $\begin{array}{c}\text { Lateral } \\
\text { Resolution } \\
\text { at 8 cm } \\
\text { for Solo }\end{array}$ & 0.20 & 0.35 & 0.34 & 0.37 \\
\hline $\begin{array}{c}\text { Axial } \\
\text { Resolution } \\
\text { at } 8 \mathrm{~cm} \\
\text { for Solo }\end{array}$ & 0.08 & 0.42 & 0.12 & 0.13 \\
\hline
\end{tabular}

\section{Spatial resolution:}

Investigation of the results of the equipment 1, an increase of the FWHM in the lateral direction is noticeable. The axial resolution and the lateral direction of the equipment 1 however, is slightly worse than the equipment 2.

\section{DISCUSSION AND CONCLUSIONS}

The Solo imaging system will detect focal lesion more than the Fucuda imaging system because the values of the spatial resolution and contrast sensitivity for Solo imaging system are better than the values of the spatial resolution and contrast sensitivity for Fucuda imaging system. This paper will hopefully contribute to a wider introduction of performance testing of medical ultrasound equipment in hospitals which, at least in Egypt, is becoming part of the equipment quality control protocol for the institutional certification.

\section{REFERENCES}

1. Archana A. Scorza, G. Lupi, S. A. Sciuto, F. Bini, F. Marinozzi "A novel to a phantom based method for maximum depth of penetration measurement in diagnostic ultrasound: a preliminary study" In: 2015 IEEE International Symposium on Medical Measurements and Applications (MEMEA 2015) Proceedings Torino, Italy, 2015, pp.369-374.

2. Tanaka S, Oshikawa O, Sasaki T, et al. "Evaluation of tissue harmonic imaging for the diagnosis of focal liver lesion." Ultrasound Med Biol. 2000; 26:183-7.

3. Tanaka S, Kitamura T, Nakanishi K, et al. Effectiveness of periodic checkup by ultrasonography for the early diagnosis of hepatocellular carcinoma. Cancer 1990; 66:2210 2214.

4. C.R. Hill (Ed.), Methods of measuring the performance of ultrasonic pulse-echo diagnostic equipment (Discussion document of International Electrotechnical Committee), Ultrasound Med. Biol. 2 (1977) 343-350. 
5. M.M. Goodsitt, P.L. Carson, S. Witt, D.L. Hykes, J.M. Kofler, Real-time B-mode ultrasound quality control test procedures, Med. Phys. 25 (1998) 1385-1406.

6. N.J. Dudley, K. Griffith, G. Houldsworth, M. Holloway, M.A. Dunn, A review of two alternative ultrasound quality assurance programs, Eur. J. Ultrasound 12 (2001) 233-245.

7. Gibson NM, Dudley NJ, Griffith K, A computerized quality control testing system for B-mode ultrasound, J. Ultrasound Med Biol. 27,1697-1711 (2001).

8. Chieko Sugawara, Akira Takahashi, Mechanical and clinical performance of pulse-inversion tissue harmonic imaging in the superficial region, J Med Ultrasonics (2010) 37:107-115

9. Thijssen JM, Wijk MC van, Cuypers MHM. Performance testing of medical ultrasound equipment. Eur J Ultrasound 2002; 15:151-164.

10. van Wijk MC, Thijssen JM. Performance testing of medical ultrasound equipment: fundamental and harmonic modes. Ultrasonics 2002; 40:585-591.

11. Thijssen JM, Weurers G, De Korte CL. Objective performance testing and quality assurance of medical ultrasound equipment. Ultrasound Med. \& Biol 2007; 33: 460-471.

12. S.W. Smith, H. Lopez, and W.J. Bodine, Frequency independent ultrasound contrast-detail analysis, J. Ultrasound Med. Bio. 1985; 11:467-477.

13. Belaid N, Cespedes I, Thijssen JM, Ophir J, Lesion detection in simulated elastographic and echographic images: A psychophysical study. J. Ultrasound Med Biol.1994; 20:877- 891.

14. Wagner RF, Insana MF, Brown DG, Unified approach to the detection and classification of speckle texture in diagnostic ultrasound, J. Opt Eng.1986; 25:738-742.

15. Thijssen JM, Oosterveld BJ, Wagner RF, Gray level transforms and lesion detectability in echographic images, J. Ultrasonic Imag.1988; 10:171-195.

16. Smith SW, Wagner RF, Sandrik JM, Lopez H, Low contrast detectability and contrast-detail analysis in medical ultrasound, IEEE Trans Sonics Ultrasonics. 1983; 30:164 -173. 